\title{
Evaluación clínica de crema facial a base de enjundia de gallina (Gallus gallus) para el tratamiento de las arrugas
}

\author{
Clinical evaluation of facial cream based in greased of hen \\ (Gallus gallus) for the treatment of the wrinkles
}

\author{
Carlos Alejandro Bell Cortez
}

http://dx.doi.org/10.21503/CienciayDesarrollo.2011.v13.02

\section{RESUMEN}

Se evaluó el efecto antiarrugas de la enjundia de gallina doméstica o gallina roja (Gallus gallus), procedente de un criadero de aves de una chacra particular, ubicada en el distrito de Chaclacayo, departamento de Lima (Perú). La grasa administrada bajo la forma de crema facial demostró propiedades para el relleno de arrugas y atenuación de líneas de expresión en el rostro. El ensayo se llevó a cabo, previo consentimiento informado, en 237 voluntarios sanos de ambos sexos, con edades mayores de 50 años. Un análisis de composición de ácidos grasos realizado por cromatografía gas-líquida en columna mostró la presencia de los siguientes ácidos: mirístico $(0,7 \%)$, palmítico (17,5\%), palmitoleico $(3,1 \%)$, hexadecatrienoico $\omega 4(0,1 \%)$, esteárico $(4,5 \%)$, oleico $\omega 7(1,8 \%)$, oleico $\omega 9(35,5 \%)$, linoleico $\omega 6$ $(31,0 \%)$, linolénico $\omega 3(1,5 \%)$, linolénico $\omega 4(0,2 \%)$, octadecatetraenoico $\omega 3(0,1 \%)$, araquídico $(0,1 \%)$, eicosenoico $\omega 9(0,3 \%)$, eicosadienoico $\omega 6(0,1 \%)$, eicosatrienoico $\omega 3(0,1 \%)$, eicosatrienoico $\omega 6(0,1 \%)$, araquidónico $\omega 6(0,2 \%)$, eicosapentaenoico $\omega 3(0,3 \%)$, docosenoico $\omega 11+9(0,1 \%)$, docosatetraenoico $\omega 3(0,1 \%)$, docosapentaenoico $\omega 3(0,1 \%)$, docosahexaenoico $\omega 3(0,2 \%)$. El ensayo de DL50 reportó gran inocuidad, y las pruebas de sensibilidad cutánea y reactividad aguda realizadas en conejos mostraron que no es irritante para la piel, por lo que la enjundia de gallina se presenta como una fuente de materia prima natural, de bajo costo, con un gran potencial para su empleo en cosmética.

Palabras clave: enjundia de gallina, efecto antiarrugas, estudio químico , cosmética.

\section{ABSTRACT}

It was evaluated the effect no wrinkles of the grease of domestic hen or red (Gallus gallus) hen, coming from a hatchery of birds of a particular vegetable garden, located in the district of Chaclacayo, department of Lima (Peru). The fat administered under the form of facial cream demonstrated properties for the filler of wrinkles and attenuation of expression en lines the face. The test was carried out, informed previous consent, in 237 healthy volunteers of both sexes, with ages bigger than 50 years. An analysis of composition of fatty acid carried out by gas-liquid cromatografía in column showed the presence of the following acids: mirístico $(0,7 \%)$, palmítico $(17,5 \%)$, palmitoleico $(3,1 \%)$, hexadecatrienoico $\omega 4(0,1 \%)$, esteárico $(4,5 \%)$, oleico, $\omega 7(1,8 \%)$, oleico $\omega 9(35,5 \%)$, linoleico $\omega 6$ $(31,0 \%)$, linolénico $\omega 3(1,5 \%)$, linolénico $\omega 4(0,2 \%)$, octadecatetraenoico $\omega 3(0,1 \%)$, araquídico $(0,1 \%)$, eicosenoico $\omega 9(0,3 \%)$, eicosadienoico $\omega 6(0,1 \%)$, eicosatrienoico $\omega 3(0,1 \%)$, eicosatrienoico $\omega 6(0,1 \%)$, araquidónico $\omega 6(0,2 \%)$, eicosapentaenoico $\omega 3(0,3 \%)$, docosenoico $\omega 11+9(0,1 \%)$, docosatetraenoico $\omega 3(0,1 \%)$, docosapentaenoico $\omega 3(0,1 \%)$, docosahexaenoico $\omega 3(0,2 \%)$. The test of DL50 reported great harmlessness, and the tests of cutaneous sensibility and sharp reactivity carried out in rabbits it showed that it is not irritating for the skin, for what the hen grease shows up like a matter source prevails natural, of low cost, with a great potential for its employment in cosmetic.

Key words: grease of hen, no wrinkles effect, study chemical, cosmetic.

* Docente de la EAP de Enfermería de la Facultad de Ciencias de la Salud de la UAP, que en esta investigación contó con la participación de las alumnas del cuarto ciclo (2010) de la especialidad de Enfermería. 


\section{INTRODUCCIÓN}

El empleo de la enjundia (llamada también popularmente infundia) de gallina (cúmulo de grasa amarilla al final de su esqueleto) para prevenir las arrugas data desde la antigüedad.

Como se sabe, las arrugas son surcos, marcas o pliegues de la piel que aparecen a lo largo de todo el cuerpo a medida que el hombre o la mujer se van haciendo mayores. Aunque responden a un proceso natural, suponen una gran preocupación para todo el mundo, puesto que son signos claros de envejecimiento. De todas las arrugas corporales, a las que más importancia damos son las que aparecen en el rostro y las que aparecen en el cuello y manos.

La búsqueda de nuevas sustancias para el rejuvenecimiento de la piel, muchas de las cuales con eficacia temporal, ha dado lugar a que grandes empresas cosméticas del mundo ensayen un sinnúmero de productos, incluso compuestos bioactivos, lo que supone la inversión de cuantiosas sumas de dinero. En ese panorama, la enjundia de gallina se presenta como un activo cosmético natural, seguro y de bajo costo.

\section{Hipótesis}

La enjundia de gallina (Gallus gallus), administrada en forma de crema tiene efecto antiarrugas sobre la piel.

\section{MATERIAL Y MÉTODO}

\section{Material}

A. Material biológico: Enjundia de gallina; conejos machos albinos de 2 a $2,5 \mathrm{~kg}$; ratones Swiss de 20 a 25 gramos (procedentes del bioterio del Instituto Nacional de Salud).

B. Reactivos: Solución acuosa de azul de Evans al $1 \%$, solución de formaldehído al $10 \%$, etanol absoluto, aceite vegetal, alcoholes grasos y preservantes.

C. Equipo: Cromatógrafo de alta resolución (HPLC), balanza analítica, equipo emulsificador de laboratorio, potenciómetro, termómetro digital.

\section{Metodología}

\section{A. Extracción de la enjundia de gallina}

Se seleccionaron 48 gallinas domésticas o gallinas rojas (Gallus gallus), de 900-950 gramos de peso y de aproximadamente un año de edad; todas igualmente alimentadas con alimento balanceado para aves. Se extrajo la enjundia de cada una de ellas y se la lavó con alcohol isopropílico. Luego se guardó toda esta sustancia en frasco estéril y se mantuvo en refrigeración hasta su empleo.

\section{B. Análisis fisicoquímico de la enjundia de gallina}

Se efectuaron los siguientes análisis físicos: aspecto, color, olor, sabor, $\mathrm{pH}$, humedad $\mathrm{y}$ densidad. Los análisis químicos realizados fueron: acidez, cenizas, grasas totales, índices de refracción, saponificación, peróxido y yodo.

\section{Análisis instrumental de la enjundia de gallina}

Se identificaron y cuantificaron ácidos grasos por cromatografía de gases.

\section{Formulación y estabilidad de la crema facial}

Luego de ensayar varias formulaciones que no solo fueran compatibles con el principio estudiado sino que, sobre todo, fueran estables en el tiempo, se eligió aquella que a la vez tuviese un bajo costo y cumpliese con los siguientes requisitos: 


\begin{tabular}{lc}
\hline Enjundia de gallina (Gallus gallus) & $20,00 \%$ \\
Alcoholes grasos & $15,20 \%$ \\
\hline Agentes saponificantes & $5,50 \%$ \\
Conservadores & $0,20 \%$ \\
\hline Agua destilada estéril & $59,10 \%$ \\
\hline
\end{tabular}

\section{E. Ensayo de estabilidad acelerada de la formulación.}

Sin duda, una crema bien formulada debe satisfacer diversos criterios, y entre ellos, el poseer una buena estabilidad física, porque sin ella, cualquier emulsión prontamente se separa en dos fases. Por ello, con el fin de determinar la estabilidad del preparado y controlar sus posibles alteraciones, se efectuaron ensayos de estabilidad acelerada, sometiendo la formulación a diferentes temperaturas. Para realizar esta prueba, se procedió a evaluar cada quince días, durante tres meses, los siguientes parámetros del producto: aspecto, consistencia, color, olor y $\mathrm{pH}$.

\section{F. Ensayo de irritabilidad en conejos}

Se realizó por el método de Finklestein modificado. La prueba mide la irritabilidad aditiva producida por una sustancia de prueba y un irritante primario (formaldehído). Un colorante vital, el azul de Evans, se inyectó por vía endovenosa. Este colorante se une a las proteínas circulantes y es extravasado en la piel inflamada o irritada por la acción de la sustancia de prueba. Como control positivo se empleó alcohol absoluto, y como control negativo se usó aceite vegetal.

\section{G. Toxicidad letal media (DL50)}

Se trabajó con 40 ratones albinos Swiss, los cuales fueron divididos en 8 grupos de 5 animales cada uno.

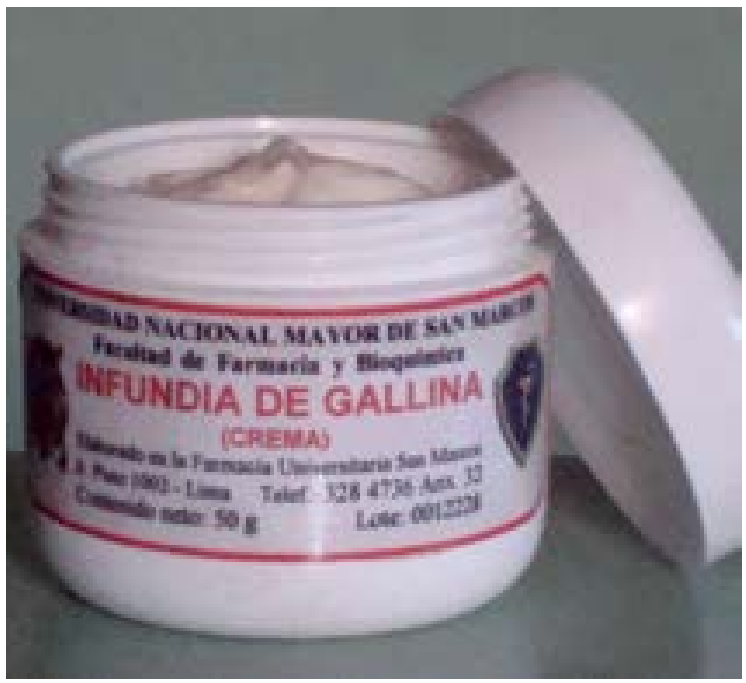

Figura 1. Producto envasado.

\section{H. Ensayo clínico efectuado en voluntarios}

Participaron en el estudio 237 voluntarios sanos de ambos sexos, con edades mayores de 50 años, residentes en diversos distritos de Lima Metropolitana.

Se dividió a los participantes en dos grupos:

- Grupo I (grupo problema, de 222 pacientes), a los que se administró crema con enjundia de gallina.

- Grupo II (grupo control, de 15 pacientes), a los que se administró crema sin enjundia de gallina.

Previo consentimiento escrito, los pacientes procedieron a la aplicación en el rostro de la crema a base de enjundia de gallina todas las noches, previo lavado facial con jabón de glicerina.

El estudio se extendió durante 30 días, durante el cual se administró una dosis de preparado de aproximadamente $1,5 \mathrm{~g}$ diarios, excluyendo la utilización de cualquier otra crema convencional. El seguimiento clínico se efectuó cada 7 días. 


\section{RESULTADOS}

\section{A. Análisis fisicoquímico de la enjundia de gallina}

Tabla 1. Protocolo de análisis de la enjundia de gallina.

\begin{tabular}{ll}
\hline PRODUCTO & $:$ Enjundia de Gallina (Gallus gallus) \\
CANTIDAD & $:$ i $120 \mathrm{~g}$ \\
FECHA DE EXTRACCIÓN & $:$ Abril del 2009 \\
FECHA DE ANÁLISIS & $:$ Abril del 2009 \\
DETERMINACIONES & RESULTADOS \\
A) FÍSICAS: & Líquido oleoso, libre de partículas extrañas \\
ASPECTO & Amarillo \\
COLOR & Insípido \\
SABOR & Sui géneris \\
OLOR & 6,6 \\
pH (25 ${ }^{\circ}$ C) & $0,7932 \%$ \\
HUMEDAD & 0,9046 \\
DENSIDAD ( 30 ${ }^{\circ}$ C) & \\
B) QUÍMICAS: & $1,398 \%$ \\
ÍNDICE DE REFRACCIÓN & $184,77 \%$ \\
ÍNDICE DE SAPONIFICACIÓN & $0,000 \%$ \\
ÍNDICE DE PERÓXIDO & $58,39 \%$ \\
ÍNDICE DE YODO & $0,36 \%$ \\
ACIDEZ (expresado en ácido oleico) & No presenta \\
CENIZAS TOTALES & $84,8766 \%$ \\
GRASAS TOTALES & \\
\hline
\end{tabular}

B. Análisis por instrumentación de la enjundia de gallina

Tabla 2. Análisis de ácidos grasos.

\begin{tabular}{lcc}
\hline PRODUCTO & $:$ Enjundia de Gallina (Gallus gallus) \\
DETERMINACIÓN & $:$ & Ácidos grasos \\
FECHA & $:$ & $23-04-2009$ \\
& $:$ & AOCS Official Method Ce 1b-89, Fifth Edition \\
MÉTODO USADO & 1997 Fatty Acid Composition by GLC \\
\multicolumn{1}{c}{ ÁCIDOS GRASOS } & $\mathrm{C}$ n:m & $\%$ \\
Ácido mirístico & $(14: 0)$ & 0,7 \\
Ácido palmítico & $(16: 0)$ & 17,5 \\
Ácido palmitoleico & $(16: 1)$ & 3,1
\end{tabular}


Ácido hexadecatrienoico $\omega_{4}$

Ácido esteárico

Ácido oleico $\omega_{7}$

Ácido oleico $\omega_{9}$

Ácido linoleico $\omega_{6}$

Ácido linolénico $\omega_{3}$

Ácido linolénico $\omega_{4}$

Ácido octadecatetraenoico $\omega_{3}$

Ácido araquídico

Ácido eicosenoico $\omega_{9}$

Ácido eicosadienoico $\omega_{6}$

Ácido eicosatrienoico $\omega_{3}$

Ácido araquidónico $\omega_{6}$

Ácido eicosapentaenoico $\omega_{3}$

Ácido docosenoico $\omega_{11+9}$

Ácido docosatetraenoico $\omega_{6}$

Ácido docosapentaenoico $\omega_{3}$

Ácido docosahexaenoico $\omega_{3}$
$(16: 3)$

(22:6)
0,1

4,5

1,8

35,5

31,0

1,5

0,2

0,1

0,1

0,3

0,1

0,1

0,2

0,3

0,1

0,1

0,1

0,2

\section{RESUMEN}

Saturados

$22,80 \%$

Monoinsaturados

$40,80 \%$

Poliinsaturados

$34,10 \%$

Total de ácidos grasos identificados

$97,70 \%$

OMEGA 3 TOTAL

$2,30 \%$

OMEGA 6 TOTAL

$31,50 \%$

$$
\begin{aligned}
& \mathrm{n}=\text { Número de carbonos } \\
& \mathrm{m}=\text { Número de enlaces } \\
& \omega=\text { Omega }
\end{aligned}
$$

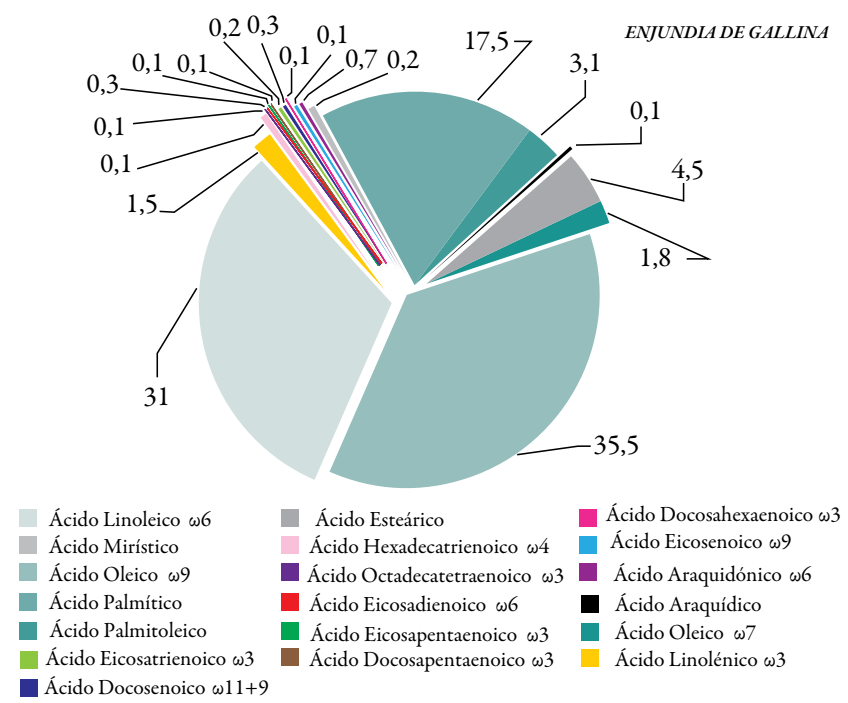

Figura 2. Análisis de ácidos grasos.

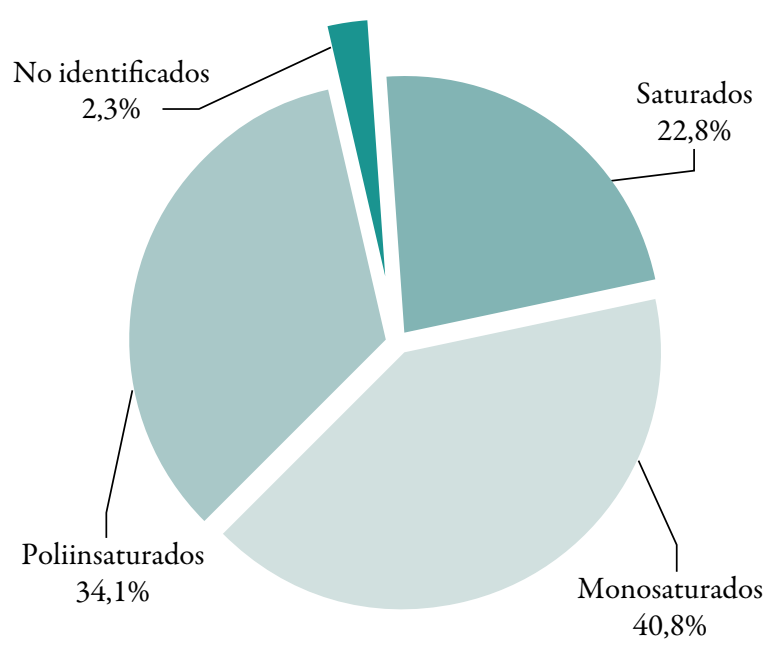

Figura 3. Resumen de ácidos grasos. 
C. Evaluación farmacotécnica de la crema facial

Tabla 3. Ficha técnica de estabilidad del producto terminado.

\begin{tabular}{|c|c|c|c|c|c|}
\hline PRODUCTO & \multicolumn{5}{|l|}{ Enjundia de gallina } \\
\hline FORMA COSMET. & \multicolumn{5}{|l|}{ Crema } \\
\hline PRESENTACIÓN & \multicolumn{5}{|l|}{ Frasco x $50 \mathrm{~g}$} \\
\hline No DE LOTE & \multicolumn{5}{|l|}{ Piloto No 001} \\
\hline FECHA FAB. & \multicolumn{5}{|l|}{ Septiembre 2009} \\
\hline FECHA VENC. & \multicolumn{5}{|l|}{ Septiembre 2012} \\
\hline \multicolumn{6}{|l|}{ RESULTADOS } \\
\hline \multirow{2}{*}{ DETERMINACIONES } & \multirow{2}{*}{ ESPECIFICACIONES } & \multicolumn{4}{|c|}{ RESULTADOS } \\
\hline & & Inicio & $1 \mathrm{mes}$ & 2 meses & 3 meses \\
\hline ASPECTO & $\begin{array}{l}\text { Emulsión tipo crema untuosa y } \\
\text { sin burbujas ni impurezas visibles. }\end{array}$ & Cumple & Cumple & Cumple & Cumple \\
\hline COLOR & Amarillenta & Cumple & Cumple & Cumple & Cumple \\
\hline OLOR & A ceras & Cumple & Cumple & Cumple & Cumple \\
\hline VISCOSIDAD & $130000-160000 \mathrm{cps}$ & 150000 & 151000 & 151000 & 151000 \\
\hline $\mathrm{pH}$ & $6,0-7,5$ & 6,8 & 6,8 & 6,8 & 6,8 \\
\hline \multicolumn{6}{|l|}{$\begin{array}{l}\text { RECUENTO } \\
\text { MICROBIANO }\end{array}$} \\
\hline AEROBIOS MESOFILOS & Menos de $100 \mathrm{ufc} / \mathrm{g}$ & Conforme & Conforme & Conforme & Conforme \\
\hline RECUENTO DE HONGOS & Menos de $10 \mathrm{ufc} / \mathrm{g}$ & Conforme & Conforme & Conforme & Conforme \\
\hline $\begin{array}{l}\text { RECUENTO DE } \\
\text { LEVADURAS }\end{array}$ & Menos de $10 \mathrm{ufc} / \mathrm{g}$ & Conforme & Conforme & Conforme & Conforme \\
\hline ESCHERICHIA COLI & Ausente en $10 \mathrm{~g}$ & Conforme & Conforme & Conforme & Conforme \\
\hline SALMONELLA s.p.p. & Ausente en $10 \mathrm{~g}$ & Conforme & Conforme & Conforme & Conforme \\
\hline $\begin{array}{l}\text { STAPHYLOCOCCUS } \\
\text { AUREUS }\end{array}$ & Ausente en $10 \mathrm{~g}$ & Conforme & Conforme & Conforme & Conforme \\
\hline $\begin{array}{l}\text { PSEUDOMONAS } \\
\text { AERUGINOSA }\end{array}$ & Ausente en $10 \mathrm{~g}$ & Conforme & Conforme & Conforme & Conforme \\
\hline
\end{tabular}

D. Prueba de sensibilidad cutánea y reactividad aguda en conejos

Tabla 4. Evaluación de la prueba de sensibilidad Cutánea.

\begin{tabular}{c|c|c|c}
\hline $\begin{array}{c}\text { SUSTANCIA DE } \\
\text { PRUEBA }\end{array}$ & $\begin{array}{c}\text { EVALUACIÓN } \\
\text { (horas) }\end{array}$ & PUNTAJE & CLASIFICACIÓN \\
\hline \multirow{2}{*}{ Enjundia de gallina } & 1 & - & No irritante \\
& 6 & - & No irritante \\
& 24 & - & No irritante \\
\hline
\end{tabular}




\section{E. Toxicidad letal media $\left(\mathrm{dl}_{50}\right)$}

El ensayo de DL50 reportó gran inocuidad. Luego de administrar una dosis promedio de hasta $3,5 \mathrm{~mL} / 20 \mathrm{~g}$ de enjundia de gallina por vía oral, los animales de experimentación permanecieron vivos.
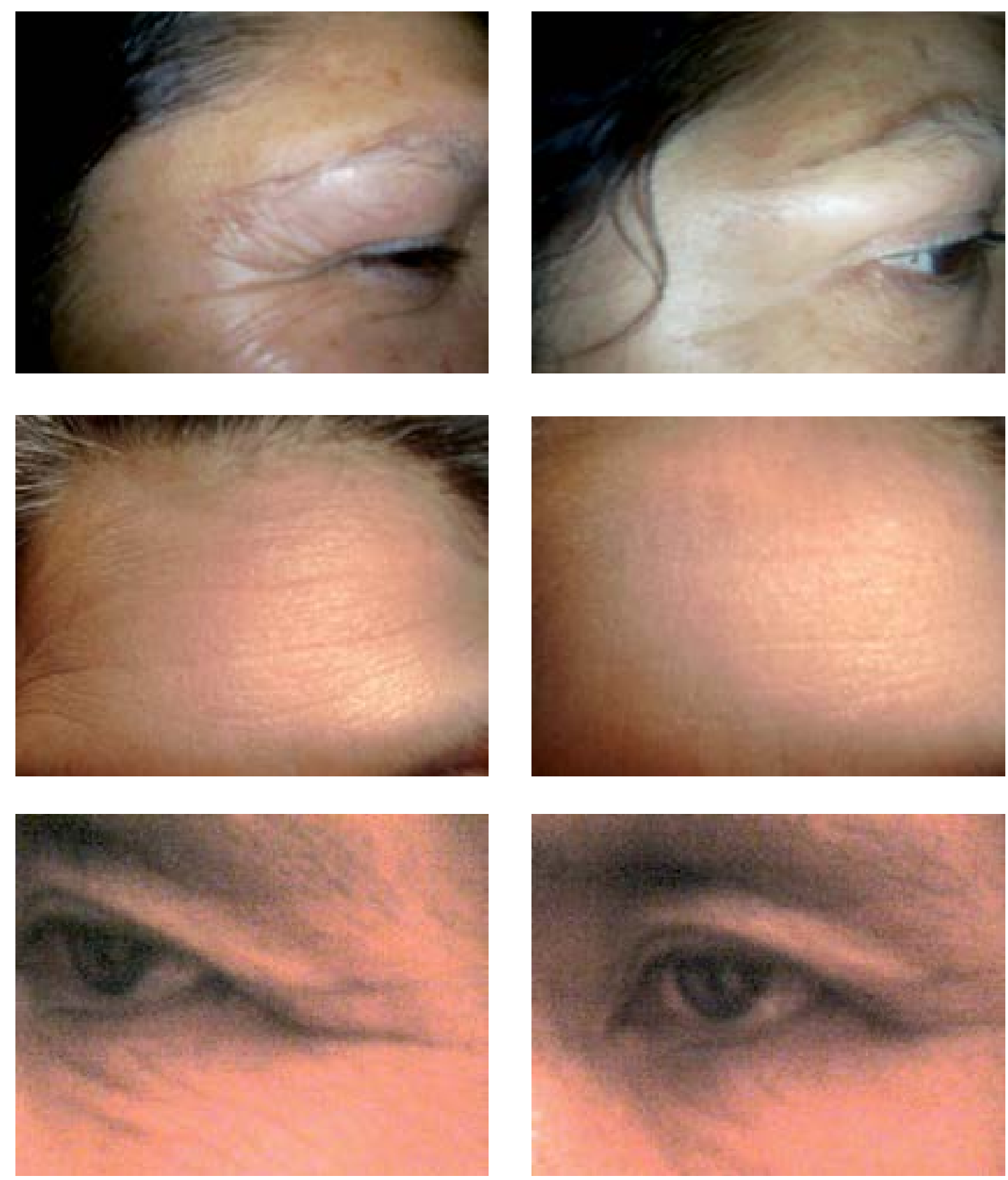

Figura 4. Evolución del relleno de arrugas y atenuación de líneas de expresión.

\section{F. Evaluación clínica}

Los resultados del comportamiento clínico a la $1^{\text {a }}, 2^{\text {a }}, 3^{\text {era }}$ y $4^{\text {ta }}$ semana de tratamiento se aprecian en la tabla 4 . Nótese que a la $1^{\text {a }}$ semana de aplicación de la crema, todos los pacientes del grupo I manifestaron apreciable mejoría, en tanto que la totalidad de pacientes del grupo II evidenciaron efectos nulos.
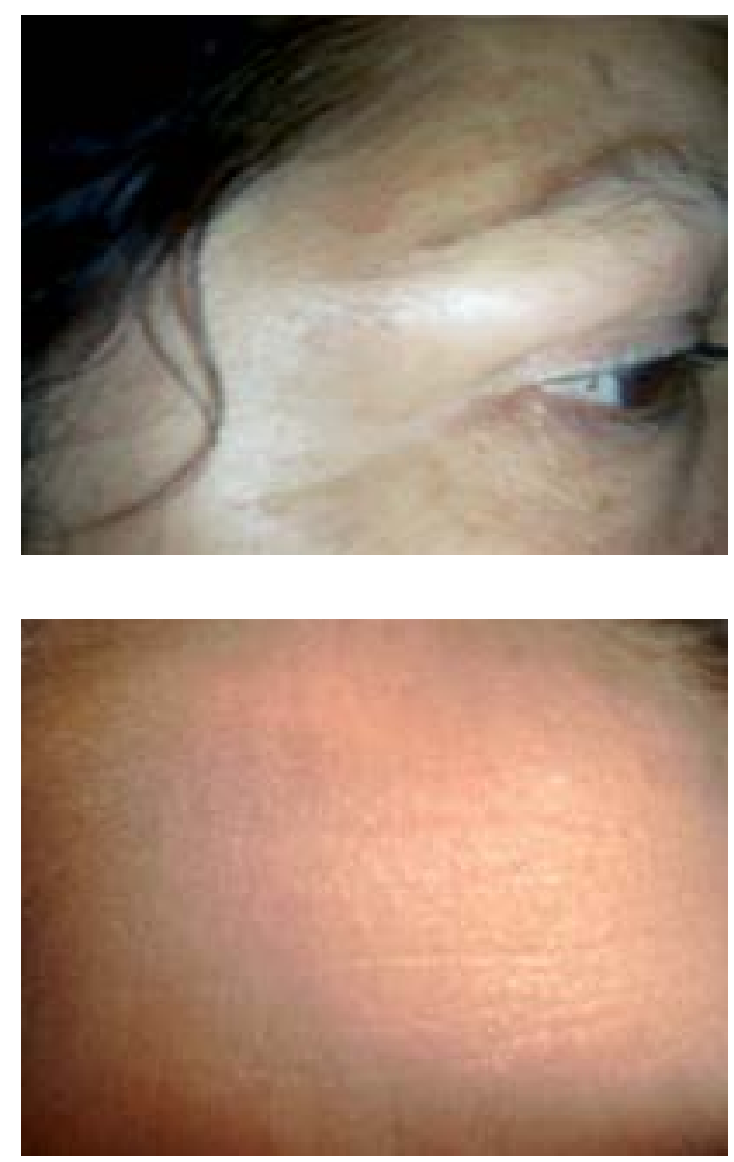

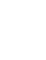




\section{Tabla 5. Valoración clínica por semanas.}

\begin{tabular}{|c|c|c|c|c|c|c|c|c|c|c|c|c|c|c|c|c|}
\hline \multirow{3}{*}{ CALIFICACIÓN } & \multicolumn{4}{|c|}{$1^{\text {a }}$ Semana } & \multicolumn{4}{|c|}{$2^{a}$ Semana } & \multicolumn{4}{|c|}{$3^{\text {era }}$ Semana } & \multicolumn{4}{|c|}{$4^{\text {ta }}$ Semana } \\
\hline & \multicolumn{2}{|c|}{ GRUPO I } & \multicolumn{2}{|c|}{ GRUPO II } & \multicolumn{2}{|c|}{ GRUPO I } & \multicolumn{2}{|c|}{ GRUPO II } & \multicolumn{2}{|c|}{ GRUPO I } & \multicolumn{2}{|c|}{ GRUPO II } & \multicolumn{2}{|c|}{ GRUPO I } & \multicolumn{2}{|c|}{ GRUPO II } \\
\hline & No & $\%$ & No & $\%$ & No & $\%$ & No & $\%$ & No & $\%$ & No & $\%$ & No & $\%$ & No & $\%$ \\
\hline Excelente & - & - & - & - & - & - & - & - & 19 & 8,56 & - & - & 37 & 16,67 & - & - \\
\hline Buena & - & - & - & - & 108 & 48,65 & - & - & 176 & 79,28 & - & - & 182 & 81,98 & - & - \\
\hline Regular & 222 & 100,0 & - & - & 114 & 51,35 & - & - & 27 & 12,16 & - & - & 3 & - & - & - \\
\hline Nula & - & - & 15 & 100,0 & - & - & 15 & 100,0 & - & - & 15 & 100,0 & - & - & 15 & 100,0 \\
\hline TOTAL & 222 & 100,0 & 15 & 100,0 & 222 & 100,0 & 15 & 100,0 & 222 & 100,0 & 15 & 100,0 & 222 & 100,0 & 15 & 100,0 \\
\hline
\end{tabular}

En consecuencia, de acuerdo con la tabla calificadora de resultados de Balbuena Gonzales y Chapano Jairo (1987), la evaluación clínica corresponde a la clasificación que se señala en la tabla 5:

\section{Tabla 6. Evaluación clínica según clasificación.}

\begin{tabular}{lc|c}
\multicolumn{1}{c|}{ CALIFICACIÓN } & No casos & $\%$ \\
$\begin{array}{lcc}\text { EXCELENTE } \\
\text { (Reducción entre 80-100\%) }\end{array}$ & 37 & 16,67 \\
$\begin{array}{l}\text { BUENA } \\
\text { (Reducción entre el 60-80\%) }\end{array}$ & 182 & 81,98 \\
$\begin{array}{l}\text { REGULAR } \\
\text { (Reducción entre 40-60\%) }\end{array}$ & 3 & 1,35 \\
$\begin{array}{l}\text { NULA } \\
\text { (Reducción menor de un 40\%) }\end{array}$ & - & - \\
\cline { 1 - 2 } SUBTOTAL & 222 & 100 \\
\hline GRUPO CONTROL & 15 & \\
\cline { 1 - 2 } TOTALES & 237 & \\
\hline
\end{tabular}

\section{ANÁLISIS Y DISCUSIÓN}

Los resultados obtenidos muestran una marcada acción favorable de la enjundia de gallina en el relleno de arrugas y atenuación de líneas de expresión (figura 2, tabla 4 y tabla 5).

Durante la evaluación semanal, se observó que no hubo alteraciones visibles en la piel, salvo la suavidad que proporcionaba la crema a ambos grupos de estudio, y que era del agrado de los pacientes.

Fisicoquímicamente (tablas 1,2 y figuras 1 y2), la enjundia de gallina demostró tener un alto porcentaje de ácidos grasos (22,8\% de saturados, $40,8 \%$ de monoinsaturados y $34,1 \%$ de poliinsaturados), lo que indica que su composición en ácidos grasos (especialmente en omegas 3 y 6) es la característica química más importante de esta sustancia.

Al evaluar la toxicidad de la enjundia de gallina, se halló un DL50 por encima de 160 $000 \mathrm{mg} / \mathrm{kg}$ en ratones, lo que, según la escala de Williams y Burson, la hace inocua, al no haber producido ningún tipo de problema en los animales de experimentación, en comparación con el grupo control hasta las 48 horas. 
El estudio de la prueba de sensibilidad cutánea y reactividad aguda (tabla 6) llevado a cabo con la enjundia de gallina en conejos brindó información de su NO IRRITABILIDAD, lo que permite afirmar que la enjundia puede ser empleada tópicamente, sin riesgo de producir reacciones alérgicas.

Finalmente, en el estudio clínico llevado a cabo con la enjundia de gallina en forma de crema (figura 1) se observó una recuperación perceptible de las arrugas y las líneas de expresión (figura 2), no presentándose durante el ensayo manifestaciones de prurito ni alergias en el sitio de aplicación, por lo que se puede afirmar, en términos generales, que el ensayo tuvo una evolución satisfactoria.

\section{CONCLUSIONES}

1. La enjundia de gallina (Gallus gallus), administrada bajo la forma de crema facial, mostró tener propiedades para el relleno de arrugas y la atenuación de líneas de expresión.

2. Por su fácil adquisición, bajo costo y ser una sustancia de origen natural que ha demostrado ser inocua, no irritante $\mathrm{y}$ totalmente estable, la enjundia de gallina puede constituir un importante activo para aplicación en cosmética y dermatología.

\section{REFERENCIAS BIBLIOGRÁFICAS}

1. Williams P., Burson J. Industrial Toxicology Safety and Health Applications in the Work Place. New York, Edit de Van Norstrand Rein Hold Company, 1985 p. 45-49.

2. Coks L., Rede V. Laboratory Handbook for Oil and fat Analysis Academic Press. London, N.Y., 1986.

3. Association of Official and Analytical Chemest's Society (AOAC). Standards Methods of Analysis. $14^{\text {th }}$ Edition, Washington D.C., 1990.

4. Draize J. "Methods to the Study of the Irritation and Toxicity of Substances Applied Topically to the Skin and Membranes". $J$ Pharm. 1994, 2(3): 8-12

5. ISO 10993-10. Biological Evaluation of Medical Devices. Part 10: Test for Irritation and Sensitization. European Commitee for Standarization, 1996. 